\title{
INCIDENCIA DELICTIVA: COORDINACIÓN POLICIAL ¿QUÉ ESPERAR DEL MANDO ÚNICO?
}

José García Gómez ${ }^{1}$

Resumen. El mando único policial, como antes la coordinación, levanta amplias expectativas. Pero ¿qué podemos esperar? La estadística descriptiva nos indica que no es sensato esperar reducción delictiva. No obstante, se cree pertinente hacer pruebas estadísticas más sólidas. Este trabajo hace inferencia acerca de la diferencia entre las medias delictivas en dos grupos de estados. Uno con mando único y/o coordinación policial o mayor cantidad de atenciones llevadas a cabo por la policía estatal. El segundo, con aquellos en los cuales no se observa lo especificado anteriormente. El resultado es que la incidencia delictiva media en ambos grupos es similar.

Palabras clave: incidencia delictiva, delito, victimización, estadística delictiva, seguridad pública.

Abstract. The single police command, as before the coordination, raises wide expectations. But what can we expect? Descriptive statistics indicate that it is not wise to expect criminal reduction. However, it's considered appropriate to make more robust statistical tests. This paper makes an inference about the difference between criminal means in two groups of states. One with those with presumed single command or police coordination or greater amount of attention conducted by state police. Another, with those in which aren't satisfied the conditions of the first. The result is that the average of crime incidence in both groups is statistically similar.

Keywords: crime incidence, crime, victimization, criminal statistics, public security.

\footnotetext{
${ }^{1}$ Profesor de tiempo completo nivel B en la Facultad de Economía y Relaciones Internacionales de Universidad Autónoma de Baja California (UABC), tiene Perfil Deseable PROMEP y es miembro del Sistema Nacional de Investigadores Nivel I. Es Ingeniero Civil con especialidad en Obras Urbanas por el Instituto Tecnológico de Tijuana (1990), Maestro en Administración Integral del Ambiente por el Colegio de la Frontera Norte (1998) y Doctor en Estudios del Desarrollo Global por la U.A.B.C (2008). Correo: garcia.jose39@uabc.edu.mx
} 


\section{Introducción}

En la actualidad el mando único policial en los estados, como antes lo hizo la coordinación, levanta amplias expectativas de éxito en el combate a la inseguridad pública. Por ejemplo, la visión de la Conferencia Nacional de Gobernadores (Conago); expresada por su líder, Erubiel Ávila Villegas, el gobernador del Estado de México, es que en el combate a la inseguridad, es mejor tener treinta y dos policías estatales que tratar de componer los más de mil ochocientos cuerpos policiacos municipales, que son con los que cuenta el país actualmente.

Más de mil ochocientas corporaciones policíacas municipales con dificultades económicas, de información, certificación y de control de confianza. Lo anterior, al entregar recientemente la Conago, mediante comisión integrada por quince gobernadores y el jefe de gobierno del Distrito Federal, presentó ante la mesa directiva del Senado de la República una propuesta para la implementación de la Policía Única Estatal (Becerra, 2016).

Esa perspectiva es compartida, aunque con matices, por distintos actores políticos y organizaciones partidistas. Por ejemplo desde el Partido de la Revolución Democrática (PRD), el Presidente de la Comisión Permanente del Congreso, el diputado Jesús Zambrano, al reiniciar las actividades de esa Comisión instó a sus compañeros legisladores a evitar entrampamientos en posiciones irreductibles y en su caso, dictaminar de manera simultánea las iniciativas ya presentadas sobre el tema así como las que diversas bancadas estén por presentar formalmente (Damian y Venegas, 2016).

Esa llamada a la premura en la dictaminación sin dilaciones que hace el legislador Zambrano está impulsada por una visión que percibe la necesidad de establecer una coordinación para hacer más efectiva y eficaz la acción de los cuerpos policiacos de todo el país, para reducir los índices de inseguridad (Damian y Venegas, 2016). Si bien matiza, particularmente en el combate al crimen organizado y añade, urge que se tomen decisiones al respecto, la resolución no debe aplazarse más.

En el caso del Partido Acción Nacional (PAN), la perspectiva parece ser similar. Señala su coordinador en la Cámara de Diputados, Marko Cortez, estar de acuerdo con el mando único, a condición que sea de naturaleza mixta y subsidiaria. Es decir un mando único flexible con policías estatales únicamente donde resulte necesario teniendo tanta policía 
como sea posible. Lo anterior con intención de evitar la concentración de poder en los gobernadores (Rosas, 2016).

Respecto al Partido Revolucionario Institucional (PRI) si bien todavía hasta hace unos pocos días no se observaba una postura clara, por un lado su coordinador en la cámara de diputados César Camacho declaraba, habrá mando único, su líder en el senado Emilio Gamboa señalaba, no se ha definido si vamos por el mando único o no (Damian y Venegas, 2016b). En su reciente convención de Querétaro el coordinador del grupo Legislativo Mauricio Ortiz Proal, afirmo: sin duda alguna vamos por el mando único y esperar en el Congreso Federal un pronto acuerdo en el tema (Vilchis, 2016).

La visión expresada por el señalado coordinador del Partido Revolucionario Institucional es similar a la expuesta por distintos actores: avanzar en la consolidación de los cuerpos policiacos, la no viabilidad de buen servicio de seguridad a partir de múltiples policías, imposibilidad de profesionalización, etc., menos cuerpos policíacos, más policías mejor preparadas, posibilidad de varias estrategias en términos operativos más eficientes, en resumen, menos cuerpos policiacos para que se pueda trabajar mejor (Vilchis, 2016).

No obstante, realmente ¿Qué es lo que racionalmente podemos esperar del mando único o la coordinación policial? ¿Mayor eficiencia y por lo tanto reducción de la incidencia delictiva nacional?

La revisión de la estadística descriptiva delictiva nacional y por estados, nos indica que no es racional esperar reducción de la incidencia delictiva. En los últimos años en varios de los estados del país presumiblemente se ha fortalecido la referida coordinación policial o francamente instaurado el mando único; que pudiera considerarse una coordinación extrema.

No obstante, la incidencia delictiva en el país, no se ha reducido. De acuerdo a datos de la Encuesta Nacional de Victimización y Percepción sobre Seguridad Pública de INEGI, en los últimos cinco años, de 2010 a 2014, la incidencia delictiva nacional se ha recrudecido.

Los delitos por cada cien mil habitantes se incrementaron en poco más del treinta y seis por ciento, de treinta mil quinientos treinta y cinco en 2010, llegó la cifra hasta cuarenta y un mil seiscientos cincuenta y cinco en 2014. Con un mínimo de veinte y nueve mil doscientos en 2012 y un máximo de los cuarenta y un mil seiscientos cincuenta y cinco en 2014, el último año con información (ver cuadro 1) y una desviación estándar promedio anual de diez mil trescientos setenta y tres delitos en el periodo señalado. 
Por estado, tenemos el ejemplo de Guerrero, en el cual pareciera existir coordinación y/o mando único, a partir de la intervención del gobierno federal y la instrumentación de diversos programas de seguridad. En los últimos años se han implementado dos grandes planes, el Plan Nuevo Guerrero y más recientemente el plan para un Guerrero Prospero e incluyente.

Se han movilizado tropas del ejército, de la Marina, contingentes de la Policía Federal. Se han invertido más de cuarenta mil millones de pesos y detenido a alcaldes, policías locales, cabecillas de bandas, sicarios, etc., y todo parece ir peor (Puig, 2016). A pesar de todo lo llevado a cabo, lejos de reducirse la actividad delictiva se ha incrementado en más del veinte y siete por ciento de 2010 a 2014, de acuerdo a datos de la Encuesta Nacional de Victimización y Percepción sobre Seguridad Pública de INEGI.

En el noroeste del país tenemos el caso de Baja California, en el cual en el pasado reciente se observó mando único y en la actualidad, se declara con suficiencia, existe efectiva coordinación policial. No obstante, de acuerdo a la misma Encuesta Nacional de Victimización y Percepción sobre Seguridad Pública de INEGI, el estado tiene un incremento delictivo del setenta y ocho por ciento en los últimos cinco años con cincuenta y seis mil seiscientos treinta y dos delitos por cada cien mil habitantes en 2014 (Cuadro 1).

Igualmente, en el centro del país está el caso del Distrito Federal, ostensiblemente una entidad con mando único, pero en la cual no sólo se padece un incremento de la actividad delictiva en los últimos cinco años de más del treinta y cinco por ciento, también es la segunda entidad con mayor incidencia delictiva, únicamente por detrás del Estado de México, que tiene ochenta y tres mil quinientos sesenta y seis delitos por cada cien mil habitantes, contra los cincuenta y nueve mil quinientos cuarenta y cinco del primero, ambos en 2014 (cuadro 1). 


\begin{tabular}{|c|c|c|c|c|c|}
\hline \multicolumn{6}{|c|}{$\begin{array}{l}\text { Cuadro 1: Tasa de incidencia delictiva nacional y por entidad federativa } \\
\text { (ocurrencia por cada cien mil habitantes). }\end{array}$} \\
\hline \multirow{2}{*}{ Entidad } & \multicolumn{5}{|c|}{ Tasa } \\
\hline & 2010 & 2011 & 2012 & 2013 & 2014 \\
\hline Nacional & 30,535 & 29,200 & 35,139 & 41,563 & 41,655 \\
\hline Aguascalientes & 56,089 & 25,511 & 32,368 & 24,711 & 39,453 \\
\hline Baja California & 31,791 & 29,446 & 39,297 & 57,066 & 56,632 \\
\hline Baja California Sur & 25,779 & 28,884 & 31,049 & 23,747 & 34,700 \\
\hline Campeche & 20922 & 21704 & 29097 & 30597 & 29306 \\
\hline Coahuila & 29279 & 26558 & 17870 & 25451 & 18318 \\
\hline Colima & 17343 & 22287 & 25169 & 26309 & 30535 \\
\hline Chiapas & 15028 & 13663 & 12827 & 19215 & 19160 \\
\hline Chihuahua & 41903 & 30562 & 35952 & 31669 & 24295 \\
\hline Distrito Federal & 44055 & 40790 & 49198 & 51786 & 59545 \\
\hline Durango & 23803 & 21540 & 27631 & 22512 & 30080 \\
\hline Guanajuato & 23365 & 26705 & 34391 & 34110 & 40737 \\
\hline Guerrero & 33467 & 27040 & 33762 & 35366 & 42690 \\
\hline Hidalgo & 22662 & 25106 & 21874 & 23468 & 23211 \\
\hline Jalisco & 32980 & 29351 & 49083 & 47278 & 43076 \\
\hline Estado de México & 32958 & 40416 & 56752 & 93003 & 83566 \\
\hline Michoacán & 15469 & 24346 & 24362 & 25126 & 26340 \\
\hline Morelos & 28491 & 25775 & 35750 & 36524 & 43584 \\
\hline Nayarit & 31741 & 28751 & 26006 & 26609 & 32936 \\
\hline Nuevo León & 38136 & 28516 & 37076 & 32552 & 28720 \\
\hline Oaxaca & 25193 & 20991 & 18009 & 20749 & 29073 \\
\hline Puebla & 23946 & 29350 & 27318 & 31662 & 32690 \\
\hline Querétaro & 19516 & 22860 & 27197 & 27975 & 31572 \\
\hline Quintana Roo & 41093 & 37725 & 40279 & 35245 & 41381 \\
\hline San Luis Potosí & 30827 & 33878 & 35124 & 39558 & 41384 \\
\hline Sinaloa & 34254 & 29838 & 33231 & 30287 & 29139 \\
\hline Sonora & 46774 & 39029 & 34126 & 31155 & 26384 \\
\hline Tabasco & 32185 & 21357 & 24368 & 32037 & 29508 \\
\hline Tamaulipas & 27083 & 20645 & 25255 & 19417 & 33414 \\
\hline Tlaxcala & 26065 & 22387 & 18530 & 26660 & 33700 \\
\hline Veracruz & 19867 & 22579 & 23411 & 28101 & 20832 \\
\hline Yucatán & 37647 & 16599 & 22945 & 23728 & 31857 \\
\hline Zacatecas & 29688 & 18772 & 20506 & 27290 & 30058 \\
\hline
\end{tabular}


No obstante lo ya descrito, se tiene el convencimiento que bien vale la pena hacer pruebas estadísticas más sólidas respecto a la incidencia delictiva y la efectividad del mando único y/o coordinación policial.

\section{Metodología}

En razón de lo señalado, en el presente trabajo se lleva a cabo inferencia estadística acerca de la diferencia entre las medias delictivas en dos grupos de estados. El primer grupo formado con aquellos estados con presumible mando único y/o coordinación policial o en su caso con mayor cantidad de atenciones, por hechos presuntamente delictivos, llevadas a cabo por la policía estatal.

El segundo, con aquellos estados en los cuales no se presume coordinación policial y/o mando único ni se tiene mayor porcentaje de atenciones policiacas, presumiblemente por hechos delictivos, llevadas a cabo por la corporación policíaca estatal.

\section{Hipótesis de trabajo}

La anteriormente buscada y alabada coordinación policiaca y/o el actual exaltado mando único no reducen la incidencia delictiva. Estadísticamente la incidencia delictiva media estatal en México es igual bajo coordinación policial o mando único que sin esta circunstancia de trabajo policial.

\section{Planteamiento general}

Como la idea y objetivo general es determinar si estadísticamente la coordinación policial o mando único reducen la incidencia delictiva, se trabaja con los datos que respecto a la tasa de incidencia delictiva por entidad federativa por cada cien mil habitantes, proporciona el INEGI. Esta base de datos será dividida en dos grupos: estados en los cuales se presume mando único y/o trabajo policial coordinado o tienen mayor cantidad de atenciones, presumiblemente por hechos delictivos, llevadas a cabo por la policía estatal (Cuadro 2) y los estados en los cuales la mayor cantidad de atenciones las lleva a cabo la policía municipal y en los cuales tampoco se presume coordinación policial y/o mando único (Cuadro 3). 
Cuadro 2: Tasa de incidencia delictiva por entidad federativa de ocurrencia, con presumible mando único y/o coordinación policial o con mayor cantidad de atenciones, presumiblemente por hechos delictivos, llevadas a cabo por la policía estatal, por cada cien mil habitantes.

\begin{tabular}{|l|r|r|r|r|r|}
\hline \multirow{2}{*}{ Entidad } & \multicolumn{5}{|c|}{ Tasa } \\
\cline { 2 - 6 } & \multicolumn{1}{|c|}{2010} & \multicolumn{1}{c|}{2011} & \multicolumn{1}{c|}{2012} & \multicolumn{1}{c|}{2013} & \multicolumn{1}{c|}{2014} \\
\hline Aguascalientes & 56,089 & 25,511 & 32,368 & 24,711 & 39,453 \\
\hline $\begin{array}{l}\text { Baja } \\
\text { California }\end{array}$ & 31,791 & 29,446 & 39,297 & 57,066 & 56,632 \\
\hline Jalisco & 32,980 & 29,351 & 49,083 & 47,278 & 43,076 \\
\hline $\begin{array}{l}\text { Michoacán de } \\
\text { Ocampo }\end{array}$ & 15,469 & 24,346 & 24,362 & 25,126 & 26,340 \\
\hline Nuevo León & 38,136 & 28,516 & 37,076 & 32,552 & 28,720 \\
\hline Morelos & 28,491 & 25,775 & 35,750 & 36,524 & 43,584 \\
\hline Tamaulipas & 27,083 & 20,645 & 25,255 & 19,417 & 33,414 \\
\hline Hidalgo & 22,662 & 25,106 & 21,874 & 23,468 & 23,211 \\
\hline Colima & 17,343 & 22,287 & 25,169 & 26,309 & 30,535 \\
\hline Guerrero & 33,467 & 27,040 & 33,762 & 35,366 & 42,690 \\
\hline Campeche & 20,922 & 21,704 & 29,097 & 30,597 & 29,306 \\
\hline Nayarit & 31,741 & 28,751 & 26,006 & 26,609 & 32,936 \\
\hline $\begin{array}{l}\text { Distrito } \\
\text { Federal }\end{array}$ & 44,055 & 40,790 & 49,198 & 51,786 & 59,545 \\
\hline $\begin{array}{l}\text { Fuente: elaboración propia con datos de la Encuesta Nacional de Victimización y } \\
\text { Percepción sobre Seguridad Pública de INEGI. }\end{array}$ & & & \\
\hline
\end{tabular}




\begin{tabular}{|c|c|c|c|c|c|}
\hline \multicolumn{6}{|c|}{$\begin{array}{l}\text { Cuadro 3: Tasa de incidencia delictiva por entidad federativa de } \\
\text { ocurrencia, sin presumible mando único y/o coordinación policial, por } \\
\text { cada cien mil habitantes. }\end{array}$} \\
\hline \multirow{2}{*}{ Entidad } & \multicolumn{5}{|c|}{ Tasa } \\
\hline & 2010 & 2011 & 2012 & 2013 & 2014 \\
\hline $\begin{array}{l}\text { Coahuila } \\
\text { de } \\
\text { Zaragoza }\end{array}$ & 29,279 & 26,558 & 17,870 & 25,451 & 18,318 \\
\hline Chiapas & 15,028 & 13,663 & 12,827 & 19,215 & 19,160 \\
\hline Chihuahua & 41,903 & 30,562 & 35,952 & 31,669 & 24,295 \\
\hline Durango & 23,803 & 21,540 & 27,631 & 22,512 & 30,080 \\
\hline Guanajuato & 23,365 & 26,705 & 34,391 & 34,110 & 40,737 \\
\hline $\begin{array}{l}\text { Estado de } \\
\text { México }\end{array}$ & 32,958 & 40,416 & 56,752 & 93,003 & 83,566 \\
\hline Oaxaca & 25,193 & 20,991 & 18,009 & 20,749 & 29,073 \\
\hline Puebla & 23,946 & 29,350 & 27,318 & 31,662 & 32,690 \\
\hline Querétaro & 19,516 & 22,860 & 27,197 & 27,975 & 31,572 \\
\hline $\begin{array}{l}\text { Quintana } \\
\text { Roo }\end{array}$ & 41,093 & 37,725 & 40,279 & 35,245 & 41,381 \\
\hline $\begin{array}{l}\text { San Luis } \\
\text { Potosí }\end{array}$ & 30,827 & 33,878 & 35,124 & 39,558 & 41,384 \\
\hline Sinaloa & 34,254 & 29,838 & 33,231 & 30,287 & 29,139 \\
\hline Sonora & 46,774 & 39,029 & 34,126 & 31,155 & 26,384 \\
\hline Tabasco & 32,185 & 21,357 & 24,368 & 32,037 & 29,508 \\
\hline Tlaxcala & 26,065 & 22,387 & 18,530 & 26,660 & 33,700 \\
\hline $\begin{array}{l}\text { Veracruz } \\
\text { de Ignacio } \\
\text { de la Llave }\end{array}$ & 19,867 & 22,579 & 23,411 & 28,101 & 20,832 \\
\hline Yucatán & 37,647 & 16,599 & 22,945 & 23,728 & 31,857 \\
\hline Zacatecas & 29,688 & 18,772 & 20,506 & 27,290 & 30,058 \\
\hline
\end{tabular}




\section{Supuestos generales}

1. Las dos poblaciones son independientes.

2. Los datos obtenidos lo fueron en muestras aleatorias simples.

3. Las dos poblaciones tienen distribuciones normales.

Para la prueba estadística se utiliza como grados de libertad al valor más pequeño de $n_{1}-1$ y $n_{2}-1$.

\section{Supuestos del análisis y estadístico de prueba}

Hipótesis nula, $\mathrm{H} 0: \mu_{1}=\mu_{2}$

Nivel de significancia, $\alpha=0.05$.

Estadístico de prueba $\mathrm{t}=\frac{\left(x_{1}-x_{2}\right)-\left(\mu_{1}-\mu_{2}\right)}{\sqrt{\frac{s_{1}^{2}}{n_{1}}+\frac{s_{2}^{2}}{n_{2}}}}$

(fórmula 1)

Donde:

$x_{1}=$ La media muestral de la incidencia delictiva de estados en los cuales se presume mando único y/o trabajo policial coordinado o tienen mayor cantidad de atenciones, presumiblemente por hechos delictivos, llevadas a cabo por la policía estatal.

$x_{2}=$ La media muestral de la incidencia delictiva de estados en los cuales no se presume mando único y/o trabajo policial coordinado y tampoco se tiene mayor cantidad de atenciones, presumiblemente por hechos delictivos, llevadas a cabo por la policía estatal.

$\mu_{1}=$ La media poblacional de la incidencia delictiva de estados en los cuales se presume mando único y/o trabajo policial coordinado o se tiene mayor cantidad de atenciones, presumiblemente por hechos delictivos, llevadas a cabo por la policía estatal. 
$\mu_{2}=$ La media poblacional de la incidencia delictiva de estados en los cuales no se presume mando único y/o trabajo policial coordinado y tampoco se tiene mayor cantidad de atenciones, presumiblemente por hechos delictivos, llevadas a cabo por la policía estatal.

$S_{1}=$ La desviación estándar de los datos de incidencia delictiva en los estados en los cuales se presume mando único y/o trabajo policial coordinado o los mismos observan mayor cantidad de atenciones, presumiblemente por hechos delictivos, llevadas a cabo por la policía estatal.

$S_{2}=$ La desviación estándar de los datos de incidencia delictiva en los estados en los cuales no se presume mando único y/o trabajo policial coordinado y tampoco se tiene mayor cantidad de atenciones, presumiblemente por hechos delictivos, llevadas a cabo por la policía estatal.

El análisis estadístico se lleva a cabo respecto a los cinco años con datos disponibles. Valores a utilizar en el cálculo:

$n_{1}=13$.

$n_{2}=19$

$x_{1}=2010,30787 ; 2011,26867 ; 2012,32$ 946; 2013, 33601 у 2014, 37649 .

$x_{2}=2010,29430 ; 2011,26510 ; 2012,28501 ; 2013,31798$ y 2014, 33075

$s_{1}=2010,10$ 677; 2011, 4 897; 2012, 8 637; 2013, 11256 у 2014, 10776.

$s_{2}=2010,8090 ; 2011,7334: 2012,9844 ; 2013,15284$ y 2014, 13569. 


\section{Resultados}

Los resultados se presentan a continuación en el Cuadro 4:

\begin{tabular}{|l|r|r|r|r|r|}
\hline Cuadro 4: Estadístico de prueba por año. \\
\hline Elemento & 2010 & 2011 & 2012 & 2013 & 2014 \\
\hline $\begin{array}{l}\text { Estadístico } \\
\text { de prueba }\end{array}$ & 0.39 & 0.16 & 1.35 & 0.38 & 1.06 \\
\hline
\end{tabular}

De acuerdo a la metodología estadística y como los datos provienen de muestras cuyo tamaño no llega a 30 unidades, el estadístico o valor crítico será obtenido con base a una distribución t de student. De la misma manera y tal como ya se ha señalado los grados de libertad utilizados fueron 12 , el valor más pequeño de $n_{1}-1$ y $n_{2}-1$.

Con base a lo anterior, el valor critico a comparar para determinar la aceptación o rechazo de la hipótesis de prueba será:

Valor crítico 2.37 (Triola, 2004).

De la misma manera y puesto que el estadístico de prueba es menor en todos los casos al valor crítico, no rechazamos la hipótesis de medias poblacionales iguales.

Es decir, la incidencia delictiva media en los estados en los cuales se presume mando único y/o trabajo policial coordinado o los mismos observan mayor cantidad de atenciones, presumiblemente por hechos delictivos, llevadas a cabo por la policía estatal es igual a la incidencia delictiva media en los estados en los cuales no se presume mando único y/o trabajo policial coordinado y en los mismos tampoco se observa mayor cantidad de atenciones, presumiblemente por hechos delictivos, llevadas a cabo por la policía estatal.

\section{Conclusiones}

Se ha probado estadísticamente: el mando único y/o la coordinación policial, al menos en México, no disminuye la incidencia delictiva. Pareciera que la naturaleza del fenómeno delictivo hace insuficiente, para su disminución, el contar con la coordinación policial. 
Por ejemplo, es claro que la mayor cantidad de hechos delictivos serían responsabilidad de las fuerzas policiacas locales, son competencia del fuero común, de acuerdo a datos de 2012, del Censo Nacional de Gobierno, Seguridad Pública y Sistema Penitenciario Estatal de INEGI, el mayor porcentaje de presuntos delitos registrados en las intervenciones policiacas son de competencia del fuero común, casi el noventa y cinco por ciento de ellas contra sólo un poco menos del cinco por ciento del fuero federal.

Es quizá por lo señalado que respecto a las intervenciones policiacas, con base a los presuntos delitos registrados en las intervenciones policiacas según nivel de gobierno, el ochenta y siete por ciento lo llevan a cabo las corporaciones policiacas contra únicamente el trece por ciento por las policías estatales.

De la misma manera, antes de involucrar todos los recursos y esfuerzo en una sola perspectiva habría que tomar también en cuenta la naturaleza del sitio en el cual o en los cuales se cometen los hechos delictivos.

De acuerdo a la tasa de incidencia delictiva por cada cien mil habitantes, por tipo de delito reportada por INEGI, el delito con mayor prevalencia es el robo o asalto en la calle o transporte público (Cuadro 5). Lugares en los cuales la atribución y responsabilidad municipal es mayor.

\begin{tabular}{|l|c|}
\hline \multicolumn{2}{|l|}{$\begin{array}{l}\text { Cuadro 5: porcentaje de robo o asalto } \\
\text { en la calle o transporte público } \\
\text { respecto de la incidencia delictiva total. }\end{array}$} \\
\hline Año & \multicolumn{1}{|c|}{24.6} \\
\hline 2010 & 34.37 \\
\hline 2011 & 24.39 \\
\hline 2012 & 29.58 \\
\hline 2013 & \multicolumn{2}{|c|}{$\begin{array}{c}\text { Fuente: elaboración propia con datos de } \\
\text { INEGI respecto a la tasa de incidencia } \\
\text { delictiva por cada cien mil habitantes, por tipo } \\
\text { de delito y la Encuesta Nacional de } \\
\text { Victimización y Percepción sobre Seguridad } \\
\text { Pública de INEGI. }\end{array}$} \\
\hline
\end{tabular}

Con todo lo anterior, no se pretende afirmar que no es adecuado y conveniente tener coordinación policial. Desde luego, la misma es necesaria para instrumentar mejores 
estrategias policiacas, eficientes y eficaces. De la misma manera, es posible que la coordinación sí tenga relación directa con el combate a la delincuencia organizada, corporaciones policiacas débiles, mal capacitadas y pobremente armadas es claro que no pueden hacer frente a grupos organizados fuertemente armados. Pero es claro, a la luz de los presentes resultados que mando único y/o coordinación resultan insuficientes.

Los mismos no garantizan consolidación, profesionalización, mejor preparación, buen servicio, etc., en suma reducción de la inseguridad. De la misma manera y tal y como se está planteando bien pudiera provocar peores circunstancias, por ejemplo, desarraigo entre sociedad y sus policías. Parte del discurso referente al combate a la inseguridad señala la conveniencia de tener cuerpos policiacos cercanos a la población. La rotación y/o traslados rutinarios y periódicos tendrían efectos contrarios a la integración policía-sociedad.

Finalmente se hace notar, el flagelo de la delincuencia organizada sin duda es la que mayor atención reclama, ya que es responsable de los actos de mayor impacto: secuestros, homicidios por ajusticiamiento, tráfico de drogas a escala mayor y mediana, mayor parte de robo de autos y otros.

Sin embargo, es la delincuencia común la que en mayor medida afecta al grueso de la población: robos con violencia o sin violencia, homicidios en el marco de una violencia social multicausal, lesiones en el mismo marco ya referido y como producto de robos con violencia, delitos sexuales, daños, etc. Es en ese marco que se requiere una policía cercana a la sociedad. 


\section{Referencias}

Becerra, B. (2016) "Propone Conago a Senado elevar a rango constitucional el mando único policial" en el El Sol de México. 2 de febrero de 2016. En: $<$ http://elsoldemexico.com.mx/mexico/117265-propone-conago-a-senado-elevar-arango-contitudional-em-mando-unico-policial> [Accesado el día 18 de enero de 2016]

Damian, F y Venegas, D. (2016) "Zambrano pide reabrir debate sobre mando único" en Síntesis informativa. 8 de enero de $2016 . \quad$ En: <http://sintesis.prd.org.mx/resumen.php?articulo_id=456078> [Accesado el día 17 de enero de 2016]

Damian, F y Venegas, D. (2016b) “Coordinadores del PRI sin acuerdo por mando único” en $\begin{array}{llllll}\text { Milenio. } & 7 & \text { de } & \text { enero }\end{array}$ <http://www.milenio.com/politica/Mando_Unico_estados-mando_unico_primando_unico_congreso_0_660534261.html> [Accesado el día 15 de enero de 2016]

Garcia, I. (2016) "Mando Único: enfrenta a partidos. El destino de la reforma sobre el Mando Único podría cambiar en las próximas semanas, debido al asesinato de la alcaldesa de Temixco, Gisela Mota" en Reporte Índigo. 2 de enero de 2016. En: <http://www.reporteindigo.com/reporte/mexico/mando-unico-enfrenta-partidos> [Accesado el día 14 de enero de 2016]

INEGI. (2012) "Encuesta Nacional de Victimización y Percepción Sobre Seguridad Pública (ENVIPE) 2012" en INEGI. En: <http://www.beta.inegi.org.mx/contenidos/proyectos/enchogares/regulares/envipe/2 012/doc/resultadosenvipe2012_09.pdf > [Accesado el día 9 de enero de 2016]

INEGI. (2013) "Encuesta Nacional de Victimización y Percepción Sobre Seguridad Pública (ENVIPE) 2013” en INEGI En: <http://www.beta.inegi.org.mx/contenidos/proyectos/enchogares/regulares/envipe/2 013/doc/envipe2013_09.pdf> [Accesado el día 9 de enero de 2016]

INEGI. (2014) "Encuesta Nacional de Victimización y Percepción Sobre Seguridad Pública (ENVIPE) 2014" en INEGI. <http://www.beta.inegi.org.mx/contenidos/proyectos/enchogares/regulares/envipe/2 014/doc/envipe2014_09.pdf> [Accesado el día 9 de enero de 2016]

INEGI. (2016) "Delitos registrados. Presuntos delitos del fuero común y federal registrados en las intervenciones de la policía, por entidad federativa según el nivel de gobierno" en INEGI En: 〈http://www.beta.inegi.org.mx/temas/delitos/> [Accesado el día 8 de enero de 2016]

INEGI. (2016) "Victimización. Tasa de prevalencia delictiva por cada cien mil habitantes. Tasa total" en INEGI. En: <http://www.beta.inegi.org.mx/temas/victimizacion/> [Accesado el día 8 de enero de 2016]

Puig, C. (2016) "Guerrero se está pudriendo. Duda Razonable" en Milenio. 9 de febrero de 2016. En: <http://www.milenio.com/firmas/carlos_puig/Guerreropudriendo_18_680511982.html> [Accesado el día 9 de febrero de 2016]

Rosas, T. (2016) "PAN dice que acepta Mando Único policiaco" en El Economista. 9 de febrero de 2016. En: <http://eleconomista.com.mx/sociedad/2016/02/09/pan-diceque-acepta-mando-unico-policiaco> [Accesado el día 11 de febrero de 2016]

Triola, F. (2004) Probabilidad y Estadística. México. Pearson Educación. Novena Ed. 
Vilchis, L. (2016) “El PRI va con el mando único” en Plaza de Armas. 5 de febrero de 2016. En:

<http://www.plazadearmas.com.mx/noticias/local/2016/02/05/pri_con_mando_unic o_381229_1013.html> [Accesado el día 10 de febrero de 2016] 